\title{
What level of evidence will it take to move towards widespread adoption of transperineal prostate biopsy in the USA?
}

\author{
Sunil H. Patel $\mathbb{C}^{1} \cdot$ Christian P. Pavlovich $^{1} \cdot$ Jared S. Winoker $^{1}$ \\ Received: 13 April 2021 / Revised: 23 April 2021 / Accepted: 23 April 2021 / Published online: 10 May 2021 \\ (c) The Author(s), under exclusive licence to Springer Nature Limited 2021
}

In the recent publication, "Rationale and Protocol for Randomized Study of Transrectal and Transperineal Prostate Biopsy Efficacy and Complications (ProBE-PC study)" Mian et al. perform a focused review of the literature examining transrectal (TRBx) and transperineal (TPBx) prostate biopsy that justify a need for their ongoing randomized study in this space [1].

The authors begin by highlighting the infectious and noninfectious complications associated with TRBx, along with the high rate of post-biopsy admissions (6.9\%) and associated costs ( $>\% 15,000 /$ per admission) [2]. They review preventative strategies that have been investigated and incorporated into practice, including current standard of care strategies. With respect to non-antimicrobial methods, they emphasize povidone-iodine rectal prep as the only measure proven to reduce biopsy-associated infections. By comparison, antibiotic prophylaxis has been more rigorously studied and the authors describe the major results of targeted therapy, single versus augmented therapy, treatment duration, and data on emerging antibiotic resistance. Collectively, their findings parallel those of the American Urologic Association (AUA) guideline recommendations on antibiotic prophylaxis for TRBx [3].

The authors discuss the existing literature on magnetic resonance imaging (MRI)-targeted prostate biopsies for the detection of clinically significant prostate cancer (PCa). They note that while one systematic review found significantly greater diagnostic sensitivity with TPBx $(86 \%)$ compared to TRBx (73\%) [4], the findings of most studies suggest relatively equivalent cancer detection rates between the two biopsy approaches. A key difference between the two approaches, which has been observed in most studies,

Sunil H. Patel

Spate167@jhmi.edu

1 The Brady Urological Institute, The Johns Hopkins School of Medicine, Baltimore, MD 21287, USA is the significantly lower rate of infectious complications with TPBx $(<1 \%)$, resulting in fewer hospitalizations and death. Still, widespread adoption of the transperineal technique remains limited. The authors suggest a number of reasons for this: Conflicting study results, a lack of highquality data, pain-related issues, and a familiarity with the TRBx vs the TPBx technique,

The above summative data suggest a need for level one evidence supporting biopsy modality in men at risk of PCa. To this effect, the authors have instituted a randomized controlled trial (RCT) that is currently ongoing. In this trial, patients undergoing prostate biopsy for any reason are randomized 1:1 to undergo office-based free-hand TRBX versus TPBx under local anesthesia. Of note, a MRI is not an inclusion criterion, however, if obtained prior, men with PIRADS 3-5 lesions will have fusion targeted cores obtained before taking the 10-12 systematic/random cores. All patients will receive an enema prior to biopsy. TPBx will be performed without peri-procedural antibiotics while TRBx will be done using ciprofloxacin $500 \mathrm{mg}$ orally plus sulfamethoxazole and trimethoprim $80-160 \mathrm{mg}$ orally, $1 \mathrm{~h}$ before and $12 \mathrm{~h}$ after. A risk-adjusted group, defined as recent exposure to antibiotics or overseas travel or history of prostatitis or allergies to standard antibiotics will get Ceftriaxone (1 gm) intramuscular (IM), 1-h prior (if allergic to ceftriaxone, then gentamicin, $160 \mathrm{mg}$ IM, 1-h prior).

Primary outcome measures are 2-week and 30-day infectious complication rates. Secondary outcomes include other adverse events and cancer detection rates. To date, 320 of the target 568 patients have been enrolled, of whom 301 have completed their biopsy.

This is a superiority trial-with the underlying hypothesis that TPBx will result in fewer infectious complications than TRBX despite the omission of antibiotics in the transperineal arm, hopefully without compromising the detection of clinically significant PCa. In our institutional experience, we have been offering free-hand TPBX under local anesthesia without antibiotics for several years, and have noted infectious complications all but disappear with a 
similar saline enema/skin prep strategy. Coupling TPBx with image-fusion in the MRI era may be a game-changer for patients and clinicians alike, but there is no reason not to inform that potential with high-quality RCTs such as this and other planned comparison studies in first-time biopsy, prior negative biopsy, and active surveillance settings (NCT04815876 and a pending R01-funded trial) [5].

There is indeed an unmet need for high-quality evidence comparing the transrectal and transperineal approaches with respect to cancer detection, infection, and patient experience across a variety of biopsy indications. Findings of the study described herein will certainly add great value to the evolving prostate biopsy landscape, particularly as they pertain to infectious risks and associated procedural costs. It is worth noting that questions surrounding diagnostic accuracy between approaches, an ongoing controversial topic, may remain inadequately answered by this study. It will be important to examine the relative rates of MRI availability, MRI positivity, lesion suspicion level, targeted biopsy outcomes, and associated cancer detection rates between the trial arms. Ultimately, there should be a healthy degree of anticipation for the results of such prospective work, as it should allow us to make more definitive conclusions about the relative risks and benefits of these two biopsy approaches.

\section{Compliance with ethical standards}

Conflict of interest The authors declare no competing interests.

Publisher's note Springer Nature remains neutral with regard to jurisdictional claims in published maps and institutional affiliations.

\section{References}

1. Mian BM, Kaufman RP, Fisher HAG. Rationale and protocol for randomized study of transrectal and transperineal prostate biopsy efficacy and complications (ProBE-PC study). Prostate Cancer Prostatic Dis. 2021. https://doi.org/10.1038/s41391-021-00352-1

2. Loeb S, Carter HB, Berndt SI, Ricker W, Schaeffer EM. Complications after prostate biopsy: data from SEER-Medicare. J Urol. 2011;186:1830-4. https://doi.org/10.1016/j.juro.2011.06.057

3. Lightner DJ, Wymer K, Sanchez J, Kavoussi L. Best practice statement on urologic procedures and antimicrobial prophylaxis. $\mathrm{J}$ Urol. 2020;203:351-6. https://doi.org/10.1097/JU.000000000000 0509

4. Tu X, Liu Z, Chang T, et al. Transperineal magnetic resonance imaging-targeted biopsy may perform better than transrectal route in the detection of clinically significant prostate cancer: systematic review and meta-analysis. Clin Genitourin Cancer. 2019;17: e860-e870. https://doi.org/10.1016/j.clgc.2019.05.006

5. Hu JC, Schaeffer EM. No Title. 2021. https://clinicaltrials.gov/ct2/ show/NCT04815876. 